\title{
A new Hardy-Mulholland-type inequality with a mixed kernel
}

\section{Michael Th. Rassias ${ }^{1,2,3} \cdot$ Bicheng Yang ${ }^{4} \cdot$ Andrei Raigorodskii $^{2,5,6,7}$}

Received: 20 November 2020 / Accepted: 9 December 2020/Published online: 15 January 2021

(C) The Author(s) 2021

\begin{abstract}
By the use of weight coefficients and techniques of real analysis, we establish a new Hardy-Mulholland-type inequality with a mixed kernel and a best possible constant factor in terms of the hypergeometric function. Equivalent forms, an operator expression with the norm and reverses are also considered.
\end{abstract}

Keywords Hardy-Mulholland-type inequality · Weight coefficients · Equivalent form $\cdot$ Hypergeometric function - Operator $\cdot$ Reverse

Mathematics Subject Classification 26D15 - 47A07

Communicated by Mario Krnic.

$凶$ Michael Th. Rassias

michail.rassias@math.uzh.ch; mthrassias@yahoo.com

Bicheng Yang

bcyang@gdei.edu.cn; bcyang818@163.com

Andrei Raigorodskii

mraigor@yandex.ru

1 Institute of Mathematics, University of Zurich, 8057 Zurich, Switzerland

2 Moscow Institute of Physics and Technology, Institutskiy per, d. 9, 141700 Dolgoprudny, Russia

3 Program in Interdisciplinary Studies, Institute for Advanced Study, 1 Einstein Dr, Princeton, NJ 08540, USA

4 Department of Mathematics, Guangdong University of Education,

Guangzhou 510303, Guangdong, People's Republic of China

5 Moscow State University, 119991 Moscow, Russia 


\section{Introduction}

If $p>1, \frac{1}{p}+\frac{1}{q}=1, a_{m}, b_{n} \geq 0, a=\left\{a_{m}\right\}_{m=1}^{\infty} \in l^{p}, b=\left\{b_{n}\right\}_{n=1}^{\infty} \in l^{q}$,

$$
\|a\|_{p}=\left(\sum_{m=1}^{\infty} a_{m}^{p}\right)^{\frac{1}{p}}>0, \quad\|b\|_{q}>0
$$

then we have the following Hardy-Hilbert inequality with the best possible constant factor $\frac{\pi}{\sin (\pi / p)}$ :

$$
\sum_{m=1}^{\infty} \sum_{n=1}^{\infty} \frac{a_{m} b_{n}}{m+n}<\frac{\pi}{\sin (\pi / p)}\|a\|_{p}\|b\|_{q} .
$$

We also have the following Mulholland inequality

$$
\sum_{m=2}^{\infty} \sum_{n=2}^{\infty} \frac{a_{m} b_{n}}{\ln m n}<\frac{\pi}{\sin (\pi / p)}\left(\sum_{m=2}^{\infty} \frac{a_{m}^{p}}{m^{1-p}}\right)^{\frac{1}{p}}\left(\sum_{n=2}^{\infty} \frac{b_{n}^{q}}{n^{1-q}}\right)^{\frac{1}{q}}
$$

with the same best possible constant factor $\frac{\pi}{\sin (\pi / p)}$ (cf. [5]). The inequalities (1) and (2) are important in Mathematical Analysis and its various applications (cf. $[5,15,35-38])$.

$$
\text { If } \begin{aligned}
\mu_{i}, v_{j}>0(i, j \in \mathbf{N} & =\{1,2, \ldots\}), \\
U_{m} & :=\sum_{i=1}^{m} \mu_{i}, \quad V_{n}:=\sum_{j=1}^{n} v_{j} \quad(m, n \in \mathbf{N}),
\end{aligned}
$$

then we have the following Hardy-Hilbert-type inequality (cf. Theorem 321 of [5], replacing $\mu_{m}^{1 / q} a_{m}$ and $v_{n}^{1 / p} b_{n}$ with $a_{m}$ and $b_{n}$ ):

$$
\sum_{m=1}^{\infty} \sum_{n=1}^{\infty} \frac{a_{m} b_{n}}{U_{m}+V_{n}}<\frac{\pi}{\sin \left(\frac{\pi}{p}\right)}\left(\sum_{m=1}^{\infty} \frac{a_{m}^{p}}{\mu_{m}^{p-1}}\right)^{\frac{1}{p}}\left(\sum_{n=1}^{\infty} \frac{b_{n}^{q}}{v_{n}^{q-1}}\right)^{\frac{1}{q}} .
$$

For $\mu_{i}=v_{j}=1(i, j \in \mathbf{N})$, inequality (4) reduces to (1).

In 1998, by introducing an independent parameter $\lambda \in(0,1]$, Yang [34] proved an extension of the integral analogous of (1) with the kernel $\frac{1}{(x+y)^{\lambda}}$ for $p=q=2$. Recently, Yang [36] presented the following extensions of (1) and (2): If $\lambda_{1}, \lambda_{2} \in \mathbf{R}$, $\lambda_{1}+\lambda_{2}=\lambda, k_{\lambda}(x, y)$ is a finite non-negative homogeneous function of degree $-\lambda$, with

$$
k\left(\lambda_{1}\right)=\int_{0}^{\infty} k_{\lambda}(t, 1) t^{\lambda_{1}-1} \mathrm{~d} t \in \mathbf{R}_{+}
$$

and $k_{\lambda}(x, y) x^{\lambda_{1}-1}\left(k_{\lambda}(x, y) y^{\lambda_{2}-1}\right)$ is decreasing with respect to $x>0(y>0)$,

$$
\phi(x)=x^{p\left(1-\lambda_{1}\right)-1}, \quad \psi(x)=x^{q\left(1-\lambda_{2}\right)-1},
$$


then for $a_{m}, b_{n} \geq 0$,

$$
a=\left\{a_{m}\right\}_{m=1}^{\infty} \in l_{p, \phi}=\left\{a ;\|a\|_{p, \phi}:=\left(\sum_{m=1}^{\infty} \phi(m)\left|a_{m}\right|^{p}\right)^{\frac{1}{p}}<\infty\right\},
$$

$b=\left\{b_{n}\right\}_{n=1}^{\infty} \in l_{q, \psi},\|a\|_{p, \phi},\|b\|_{q, \psi}>0$, we have

$$
\sum_{m=1}^{\infty} \sum_{n=1}^{\infty} k_{\lambda}(m, n) a_{m} b_{n}<k\left(\lambda_{1}\right)\|a\|_{p, \phi}\|b\|_{q, \psi}
$$

where the constant factor $k\left(\lambda_{1}\right)$ is still the best possible.

Clearly, for $\lambda=1, \lambda_{1}=\frac{1}{q}, \lambda_{2}=\frac{1}{p}, k_{1}(x, y)=\frac{1}{x+y}$, the inequality (5) reduces to (1). Some other new results including multidimensional Hilbert-type inequalities, Hardy-Mulholland-type inequalities and Hardy-Hilbert-type inequalities are established in [1-4, 6-10, 12, 14, 16-31, 33, 39-48].

In this paper, by the use of weight coefficients and techniques of real analysis, we prove a new Hardy-Mulholland-type inequality with the following mixed kernel

$$
\frac{\left(\min \left\{\ln U_{m}, \ln V_{n}\right\}\right)^{\alpha}}{\left(\ln U_{m} V_{n}\right)^{\lambda+\alpha}} \quad(0 \leq-2 \alpha<\lambda \leq 2 ; m, n \in \mathbf{N} \backslash\{1\}) .
$$

and a best possible constant factor expressed in terms of the hypergeometric function. This inequality constitutes an extension of (2). Equivalent forms, operator expressions with the norm and reverses are considered as well.

\section{An example and some lemmas}

In the sequel, we consider that

$$
p \neq 0,1, \quad \frac{1}{p}+\frac{1}{q}=1, \quad \lambda_{1}+\lambda_{2}=\lambda, \quad \mu_{i}, v_{j}>0(i, j \in \mathbf{N}),
$$

with $\mu_{1}=v_{1}=1, U_{m}$ and $V_{n}$ are defined in (3), $a_{m}, b_{n} \geq 0$,

$$
\|a\|_{p, \Phi_{\lambda}}:=\left(\sum_{m=2}^{\infty} \Phi_{\lambda}(m) a_{m}^{p}\right)^{\frac{1}{p}} \text { and }\|b\|_{q, \Psi_{\lambda}}:=\left(\sum_{n=2}^{\infty} \Psi_{\lambda}(n) b_{n}^{q}\right)^{\frac{1}{q}}
$$

where

$$
\Phi_{\lambda}(m):=\frac{\left(\ln U_{m}\right)^{p\left(1-\lambda_{1}\right)-1}}{U_{m}^{1-p} \mu_{m}^{p-1}}, \quad \Psi_{\lambda}(n):=\frac{\left(\ln V_{n}\right)^{q\left(1-\lambda_{2}\right)-1}}{V_{n}^{1-q} v_{n}^{q-1}} \quad(m, n \in \mathbf{N} \backslash\{1\}) .
$$

We introduce the following hypergeometric function (cf. [32]):

$$
F(\alpha, \beta, \gamma, z):=\frac{\Gamma(\gamma)}{\Gamma(\beta) \Gamma(\gamma-\beta)} \int_{0}^{1} t^{\beta-1}(1-t)^{\gamma-\beta-1}(1-z t)^{-\alpha} \mathrm{d} t
$$


where $\operatorname{Re}(\gamma)>\operatorname{Re}(\beta)>0,|\arg (1-z)|<\pi ;(1-z t)^{-\alpha}=1$, for $z=0$.

Example 2.1 For $-\alpha<\lambda_{1}, \lambda_{2} \leq 1\left(-2 \alpha<\lambda=\lambda_{1}+\lambda_{2} \leq 2\right)$, we set

$$
k_{\lambda}(x, y):=\frac{(\min \{x, y\})^{\alpha}}{(x+y)^{\lambda+\alpha}} \quad\left((x, y) \in \mathbf{R}_{+}^{2}\right) \text {. }
$$

(1) Since $\lambda+\alpha>-\alpha$, there exists a constant $L_{\alpha}=\max \left\{2^{\alpha}, 1\right\}>0$, such that

$$
\frac{1}{(t+1)^{\lambda+\alpha}} \leq(t+1)^{\alpha} \leq L_{\alpha} \quad(t \in(0,1)) .
$$

For $\lambda_{1}, \lambda_{2}>-\alpha$, we get that

$$
\begin{aligned}
0 & <k_{\alpha}\left(\lambda_{1}\right):=\int_{0}^{\infty} k_{\lambda}(1, t) t^{\lambda_{2}-1} \mathrm{~d} t=\int_{0}^{\infty} k_{\lambda}(t, 1) t^{\lambda_{1}-1} \mathrm{~d} t \\
& =\int_{0}^{\infty} \frac{(\min \{t, 1\})^{\alpha}}{(t+1)^{\lambda+\alpha}} t^{\lambda_{1}-1} \mathrm{~d} t=\int_{0}^{1} \frac{t^{\lambda_{1}+\alpha-1}+t^{\lambda_{2}+\alpha-1}}{(t+1)^{\lambda+\alpha}} \mathrm{d} t \\
& \leq L_{\alpha} \int_{0}^{1}\left(t^{\lambda_{1}+\alpha-1}+t^{\lambda_{2}+\alpha-1}\right) \mathrm{d} t=L_{\alpha}\left(\frac{1}{\lambda_{1}+\alpha}+\frac{1}{\lambda_{2}+\alpha}\right)<\infty,
\end{aligned}
$$

and thus by (6) and (7), it follows that

$$
k_{\alpha}\left(\lambda_{1}\right)=\sum_{i=1}^{2} \frac{1}{\lambda_{i}+\alpha} F\left(\lambda+\alpha, \lambda_{i}+\alpha, \lambda_{i}+\alpha+1,-1\right) \in \mathbf{R}_{+} .
$$

(i) For $\alpha=0$, we obtain $k_{\lambda}(x, y)=\frac{1}{(x+y)^{\lambda}}(0<\lambda \leq 2)$ and

$$
k_{0}\left(\lambda_{1}\right)=\int_{0}^{\infty} \frac{t^{\lambda_{1}-1} \mathrm{~d} t}{(t+1)^{\lambda}}=B\left(\lambda_{1}, \lambda_{2}\right)=\sum_{i=1}^{2} \frac{1}{\lambda_{i}} F\left(\lambda, \lambda_{i}, \lambda_{i}+1,-1\right)
$$

(ii) for $-\alpha<\lambda+\alpha \leq 1(<2+\alpha)$, we derive that

$$
\begin{aligned}
k_{\alpha}\left(\lambda_{1}\right) & =\int_{0}^{1} \frac{t^{\lambda_{1}+\alpha-1}+t^{\lambda_{2}+\alpha-1}}{(t+1)^{\lambda+\alpha}} \mathrm{d} t \\
& =\int_{0}^{1} \sum_{k=0}^{\infty}\left(\begin{array}{c}
-\lambda-\alpha \\
k
\end{array}\right) t^{k}\left(t^{\lambda_{1}+\alpha-1}+t^{\lambda_{2}+\alpha-1}\right) \mathrm{d} t \\
& =\int_{0}^{1} \sum_{k=0}^{\infty}(-1)^{k}\left(\begin{array}{c}
\lambda+\alpha+k-1 \\
k
\end{array}\right) t^{k}\left(t^{\lambda_{1}+\alpha-1}+t^{\lambda_{2}+\alpha-1}\right) \mathrm{d} t \\
& =\int_{0}^{1} \sum_{k=0}^{\infty}\left(\begin{array}{c}
\lambda+\alpha+2 k-1 \\
2 k
\end{array}\right)\left(1-\frac{\lambda+\alpha+2 k}{2 k+1} t\right) t^{2 k}\left(t^{\lambda_{1}+\alpha-1}+t^{\lambda_{2}+\alpha-1}\right) \mathrm{d} t .
\end{aligned}
$$

\section{Since}




$$
1-\frac{\lambda+\alpha+2 k}{2 k+1} t \geq \frac{1-(\lambda+\alpha)+2 k(1-t)}{2 k+1} \geq 0,
$$

in view of the Lebesgue term by term integration theorem (cf. [13]), we obtain that

$$
\begin{aligned}
k_{\alpha}\left(\lambda_{1}\right) & =\sum_{k=0}^{\infty} \int_{0}^{1}\left(\begin{array}{c}
\lambda+\alpha+2 k-1 \\
2 k
\end{array}\right)\left(1-\frac{\lambda+\alpha+2 k}{2 k+1} t\right) t^{2 k}\left(t^{\lambda_{1}-1}+t^{\lambda_{2}-1}\right) \mathrm{d} t \\
& =\sum_{k=0}^{\infty}\left(\begin{array}{c}
-\lambda-\alpha \\
k
\end{array}\right) \int_{0}^{1} t^{k}\left(t^{\lambda_{1}+\alpha-1}+t^{\lambda_{2}+\alpha-1}\right) \mathrm{d} t \\
& =\sum_{k=0}^{\infty}\left(\begin{array}{c}
-\lambda-\alpha \\
k
\end{array}\right)\left(\frac{1}{k+\lambda_{1}+\alpha}+\frac{1}{k+\lambda_{2}+\alpha}\right) .
\end{aligned}
$$

(2) Suppose that $\alpha \leq 0(\alpha>-1)$. We have

$$
\lambda+\alpha>-\alpha \geq 0, \quad 0<\lambda_{i}+\alpha \leq \lambda_{i}(i=1,2) .
$$

For $\lambda_{2} \leq 1\left(\lambda_{2}+\alpha \leq \lambda_{2} \leq 1\right)$ and fixed $x>0$, we deduce that

$$
k_{\lambda}(x, y) \frac{1}{y^{1-\lambda_{2}}}=\left\{\begin{array}{lc}
\frac{1}{(x+y)^{\lambda+\alpha} y^{1-\left(\lambda_{2}+\alpha\right)}}, & 0<y<x \\
\frac{x^{\alpha}}{(x+y)^{\lambda+\alpha} y^{1-\lambda_{2}}}, & y \geq x
\end{array}\right.
$$

is strictly decreasing with respect to $y>0$. Similarly, for $\lambda_{1} \leq 1$ and fixed $y>0$,

$$
k_{\lambda}(x, y) \frac{1}{x^{1-\lambda_{1}}}
$$

is strictly decreasing with respect to $x>0$.

Lemma 2.2 If $0 \leq-\alpha<\lambda_{1}, \lambda_{2} \leq 1, k_{\alpha}\left(\lambda_{1}\right)$ is defined as in (7), and we define the following weight coefficients:

$$
\begin{aligned}
& \omega\left(\lambda_{2}, m\right):=\sum_{n=2}^{\infty} \frac{\left(\min \left\{\ln U_{m}, \ln V_{n}\right\}\right)^{\alpha}}{\left(\ln U_{m} V_{n}\right)^{\lambda+\alpha}} \frac{v_{n} \ln ^{\lambda_{1}} U_{m}}{V_{n} \ln ^{1-\lambda_{2}} V_{n}}, \quad m \in \mathbf{N} \backslash\{1\}, \\
& \varpi\left(\lambda_{1}, n\right):=\sum_{m=2}^{\infty} \frac{\left(\min \left\{\ln U_{m}, \ln V_{n}\right\}\right)^{\alpha}}{\left(\ln U_{m} V_{n}\right)^{\lambda+\alpha}} \frac{\mu_{m} \ln ^{\lambda_{2}} V_{n}}{U_{m} \ln ^{1-\lambda_{1}} U_{m}}, \quad n \in \mathbf{N} \backslash\{1\},
\end{aligned}
$$

then the following inequalities hold true:

$$
\begin{aligned}
& \omega\left(\lambda_{2}, m\right)<k_{\alpha}\left(\lambda_{1}\right)\left(-\alpha<\lambda_{2} \leq 1, \lambda_{1}>-\alpha ; m \in \mathbf{N} \backslash\{1\}\right), \\
& \varpi\left(\lambda_{1}, n\right)<k_{\alpha}\left(\lambda_{1}\right)\left(-\alpha<\lambda_{1} \leq 1, \lambda_{2}>-\alpha ; n \in \mathbf{N} \backslash\{1\}\right) .
\end{aligned}
$$


Proof In view of (3), we set

$$
\mu(t):=\mu_{m}, t \in(m-1, m](m \in \mathbf{N}) ; \quad v(t):=v_{n}, t \in(n-1, n](n \in \mathbf{N}),
$$

and

$$
U(x):=\int_{0}^{x} \mu(t) \mathrm{d} t(x \geq 0), \quad V(y):=\int_{0}^{y} v(t) \mathrm{d} t(y \geq 0) .
$$

Then it follows that

$$
U(m)=U_{m}, \quad V(n)=V_{n} \quad(m, n \in \mathbf{N}) .
$$

For $x \in(m-1, m)$,

$$
U^{\prime}(x)=\mu(x)=\mu_{m} \quad(m \in \mathbf{N}) ;
$$

for $y \in(n-1, n)$,

$$
V^{\prime}(y)=v(y)=v_{n} \quad(n \in \mathbf{N}) .
$$

Since $V(y)$ is strictly increasing in $(n-1, n](n \in \mathbf{N})$, and $-\alpha<\lambda_{2} \leq 1, \lambda_{1}>-\alpha$, in view of Example 2.1(2), we derive that

$$
\begin{aligned}
\omega\left(\lambda_{2}, m\right) & =\sum_{n=2}^{\infty} \int_{n-1}^{n} \frac{\left(\min \left\{\ln U_{m}, \ln V_{n}\right\}\right)^{\alpha}}{\left(\ln U_{m} V_{n}\right)^{\lambda+\alpha}} \frac{\ln ^{\lambda_{1}} U_{m}}{V_{n} \ln ^{1-\lambda_{2}} V_{n}} V^{\prime}(y) \mathrm{d} y \\
& <\sum_{n=2}^{\infty} \int_{n-1}^{n} \frac{\left(\min \left\{\ln U_{m}, \ln V(y)\right\}\right)^{\alpha}}{\left(\ln U_{m} V(y)\right)^{\lambda+\alpha}} \frac{\ln ^{\lambda_{1}} U_{m}}{V(y) \ln ^{1-\lambda_{2}} V(y)} V^{\prime}(y) \mathrm{d} y \\
& =\int_{1}^{\infty} \frac{\left(\min \left\{\ln U_{m}, \ln V(y)\right\}\right)^{\alpha}}{\left(\ln U_{m} V(y)\right)^{\lambda+\alpha}} \frac{\ln ^{\lambda_{1}} U_{m}}{V(y) \ln ^{1-\lambda_{2}} V(y)} V^{\prime}(y) \mathrm{d} y .
\end{aligned}
$$

Setting $t=\frac{\ln V(y)}{\ln U_{m}}$, we obtain that

$$
\frac{V^{\prime}(y)}{V(y)} \mathrm{d} y=\ln U_{m} \mathrm{~d} t, \quad \ln V(1)=\ln v_{1}=0\left(v_{1}=1\right)
$$

and

$$
\begin{aligned}
\omega\left(\lambda_{2}, m\right) & <\int_{0}^{\frac{\ln V(\infty)}{\ln U_{m}}} \frac{(\min \{1, t\})^{\alpha}}{(1+t)^{\lambda+\alpha}} t^{\lambda_{2}-1} \mathrm{~d} t \\
& \leq \int_{0}^{\infty} \frac{(\min \{1, t\})^{\alpha}}{(1+t)^{\lambda+\alpha}} t^{\lambda_{2}-1} \mathrm{~d} t=k_{\alpha}\left(\lambda_{1}\right) .
\end{aligned}
$$

Hence, we obtain (13). Similarly, for $-\alpha<\lambda_{1} \leq 1, \lambda_{2}>-\alpha$, we have (14).

Lemma 2.3 If $0 \leq-\alpha<\lambda_{1}, \lambda_{2} \leq 1, k_{\alpha}\left(\lambda_{1}\right)$ is defined in (7), $U_{\infty}=V_{\infty}=\infty$, there exist $m_{0}, n_{0} \in \mathbf{N}$, such that $\left\{\mu_{m}\right\}_{m=m_{0}}^{\infty}$ and $\left\{v_{n}\right\}_{n=n_{0}}^{\infty}$ are decreasing, then:

(i) for $m, n \in \mathbf{N} \backslash\{1\}$, we have 


$$
\begin{gathered}
k_{\alpha}\left(\lambda_{1}\right)\left(1-\theta\left(\lambda_{2}, m\right)\right)<\omega\left(\lambda_{2}, m\right) \quad\left(-\alpha<\lambda_{2} \leq 1, \lambda_{1}>-\alpha\right), \\
k_{\alpha}\left(\lambda_{1}\right)\left(1-\vartheta\left(\lambda_{1}, n\right)\right)<\varpi\left(\lambda_{1}, n\right) \quad\left(-\alpha<\lambda_{1} \leq 1, \lambda_{2}>-\alpha\right),
\end{gathered}
$$

where

$$
\begin{aligned}
\theta\left(\lambda_{2}, m\right) & :=\frac{1}{k_{\alpha}\left(\lambda_{1}\right)} \int_{0}^{\frac{\ln V_{n_{0}+1}}{\ln U_{m}}} \frac{(\min \{t, 1\})^{\alpha}}{(t+1)^{\lambda+\alpha}} t^{\lambda_{2}-1} \mathrm{~d} t \\
& =O\left(\frac{1}{\ln ^{\alpha+\lambda_{2}} U_{m}}\right) \in(0,1), \\
\vartheta\left(\lambda_{1}, n\right) & :=\frac{1}{k_{\alpha}\left(\lambda_{1}\right)} \int_{0}^{\frac{\ln U_{m_{0}+1}}{\ln V_{n}}} \frac{(\min \{t, 1\})^{\alpha}}{(t+1)^{\lambda+\alpha}} t^{\lambda_{1}-1} \mathrm{~d} t \\
& =O\left(\frac{1}{\ln ^{\alpha+\lambda_{1}} V_{n}}\right) \in(0,1) ;
\end{aligned}
$$

(ii) for any $a>0$, we have

$$
\begin{gathered}
\sum_{m=2}^{\infty} \frac{\mu_{m}}{U_{m} \ln ^{1+a} U_{m}}=\frac{1}{a}\left[\frac{1}{\ln ^{a} U_{m_{0}+1}}+a O_{1}(1)\right], \\
\sum_{n=2}^{\infty} \frac{v_{n}}{V_{n} \ln ^{1+a} V_{n}}=\frac{1}{a}\left[\frac{1}{\ln ^{a} V_{n_{0}+1}}+a O_{2}(1)\right] .
\end{gathered}
$$

Proof Since $v_{n} \geq v_{n+1}\left(n \geq n_{0}\right),-\alpha<\lambda_{2} \leq 1, \lambda_{1}>-\alpha$ and $V(\infty)=\infty$, by Example 2.1(2), we have

$$
\begin{aligned}
\omega\left(\lambda_{2}, m\right) & \geq \sum_{n=n_{0}+1}^{\infty} \frac{\left(\min \left\{\ln U_{m}, \ln V_{n}\right\}\right)^{\alpha}}{\left(\ln U_{m} V_{n}\right)^{\lambda+\alpha}} \frac{v_{n+1} \ln ^{\lambda_{1}} U_{m}}{V_{n} \ln ^{1-\lambda_{2}} V_{n}} \\
& >\sum_{n=n_{0}+1}^{\infty} \int_{n}^{n+1} \frac{\left(\min \left\{\ln U_{m}, \ln V(y)\right\}\right)^{\alpha}}{\left(\ln U_{m} V(y)\right)^{\lambda+\alpha}} \frac{V^{\prime}(y) \ln ^{\lambda_{1}} U_{m}}{V(y) \ln ^{1-\lambda_{2}} V(y)} \mathrm{d} y \\
& =\int_{n_{0}+1}^{\infty} \frac{\left(\min \left\{\ln U_{m}, \ln V(y)\right\}\right)^{\alpha}}{\left(\ln U_{m} V(y)\right)^{\lambda+\alpha}} \frac{V^{\prime}(y) \ln ^{\lambda_{1}} U_{m}}{V(y) \ln ^{1-\lambda_{2}} V(y)} \mathrm{d} y\left(t=\frac{\ln V(y)}{\ln U_{m}}\right) \\
& =\int_{\frac{\ln V_{n_{0}+1}}{\ln U_{m}}}^{\infty} \frac{(\min \{1, t\})^{\alpha}}{(1+t)^{\lambda+\alpha}} t^{\lambda_{2}-1} \mathrm{~d} t=k_{\alpha}\left(\lambda_{1}\right)\left(1-\theta\left(\lambda_{2}, m\right)\right)>0 .
\end{aligned}
$$

For $U_{m} \geq V_{n_{0}+1}(m \geq 2)$, by Example 2.1(1), we obtain that 


$$
\begin{aligned}
0 & <\theta\left(\lambda_{2}, m\right)=\frac{1}{k_{\alpha}\left(\lambda_{1}\right)} \int_{0}^{\frac{\ln V_{n_{0}}+1}{\ln U_{m}}} \frac{(\min \{1, t\})^{\alpha}}{(1+t)^{\lambda+\alpha}} t^{\lambda_{2}-1} \mathrm{~d} t \\
& =\frac{1}{k_{\alpha}\left(\lambda_{1}\right)} \int_{0}^{\frac{\ln V_{n_{0}+1}}{\ln U_{m}}} \frac{t^{\alpha}}{(1+t)^{\lambda+\alpha}} t^{\lambda_{2}-1} \mathrm{~d} t \\
& \leq \frac{L_{\alpha}}{k_{\alpha}\left(\lambda_{1}\right)} \int_{0}^{\frac{\ln V_{n_{0}+1}}{\ln U_{m}}} t^{\alpha+\lambda_{2}-1} \mathrm{~d} t=\frac{L_{\alpha}}{\left(\alpha+\lambda_{2}\right) k_{\alpha}\left(\lambda_{1}\right)}\left(\frac{\ln V_{n_{0}+1}}{\ln U_{m}}\right)^{\alpha+\lambda_{2}},
\end{aligned}
$$

namely, (16) and (18) follow. Similarly, for $-\alpha<\lambda_{1} \leq 1, \lambda_{2}>-\alpha$, we obtain (17) and (19).

For $a>0$, we deduce that

$$
\begin{aligned}
& \sum_{m=2}^{\infty} \frac{\mu_{m}}{U_{m} \ln ^{1+a} U_{m}}=\sum_{m=2}^{m_{0}+1} \frac{\mu_{m}}{U_{m} \ln ^{1+a} U_{m}}+\sum_{m=m_{0}+2}^{\infty} \frac{\mu_{m}}{U_{m} \ln ^{1+a} U_{m}} \\
& \quad<\sum_{m=2}^{m_{0}+1} \frac{\mu_{m}}{U_{m} \ln ^{1+a} U_{m}}+\sum_{m=m_{0}+2}^{\infty} \int_{m-1}^{m} \frac{U^{\prime}(x)}{U(x) \ln ^{1+a} U(x)} \mathrm{d} x \\
& \quad=\sum_{m=2}^{m_{0}+1} \frac{\mu_{m}}{U_{m} \ln ^{1+a} U_{m}}+\int_{m_{0}+1}^{\infty} \frac{U^{\prime}(x)}{U(x) \ln ^{1+a} U(x)} \mathrm{d} x \\
& \quad=\frac{1}{a}\left(\frac{1}{\ln ^{a} U_{m_{0}+1}}+a \sum_{m=2}^{m_{0}+1} \frac{\mu_{m}}{U_{m} \ln ^{1+a} U_{m}}\right), \\
& \sum_{m=2}^{\infty} \frac{\mu_{m}}{U_{m} \ln ^{1+a} U_{m}} \geq \sum_{m=m_{0}+1}^{\infty} \frac{\mu_{m+1}}{U_{m} \ln ^{1+a} U_{m}}>\sum_{m=m_{0}+1}^{\infty} \int_{m}^{m+1} \frac{U^{\prime}(x) \mathrm{d} x}{U(x) \ln ^{1+a} U(x)} \\
& =\int_{m_{0}+1}^{\infty} \frac{U^{\prime}(x) \mathrm{d} x}{U(x) \ln ^{1+a} U(x)}=\frac{1}{a \ln ^{a} U_{m_{0}+1}} .
\end{aligned}
$$

Hence we obtain (20). Similarly, we derive (21).

Lemma 2.4 If $0 \leq-\alpha<\lambda_{1}, \lambda_{2} \leq 1, k_{\alpha}\left(\lambda_{1}\right)$ is defined as in (7), then for

$$
0<\delta<\min \left\{\alpha+\lambda_{1}, \alpha+\lambda_{2}\right\}
$$

we have

$$
k_{\alpha}\left(\lambda_{1} \pm \delta\right)=k_{\alpha}\left(\lambda_{1}\right)+o(1)\left(\delta \rightarrow 0^{+}\right)
$$

Proof For $0<\delta<\min \left\{\alpha+\lambda_{1}, \alpha+\lambda_{2}\right\}$, we get that 


$$
\begin{aligned}
\mid k_{\alpha} & \left(\lambda_{1}+\delta\right)-k_{\alpha}\left(\lambda_{1}\right) \mid \leq \int_{0}^{\infty} \frac{(\min \{t, 1\})^{\alpha} t^{\lambda_{1}-1}\left|t^{\delta}-1\right|}{(t+1)^{\lambda+\alpha}} \mathrm{d} t \\
& =\int_{0}^{1} \frac{t^{\alpha+\lambda_{1}-1}\left(1-t^{\delta}\right)}{(t+1)^{\lambda+\alpha}} \mathrm{d} t+\int_{0}^{1} \frac{t^{\alpha+\lambda_{2}-1}\left(t^{-\delta}-1\right)}{(t+1)^{\lambda+\alpha}} \mathrm{d} t \\
& \leq L_{\alpha}\left[\int_{0}^{1} t^{\alpha+\lambda_{1}-1}\left(1-t^{\delta}\right) \mathrm{d} t+\int_{0}^{1} t^{\alpha+\lambda_{2}-1}\left(t^{-\delta}-1\right) \mathrm{d} t\right] \\
& =L_{\alpha}\left[\frac{1}{\alpha+\lambda_{1}}-\frac{1}{\alpha+\lambda_{1}+\delta}+\frac{1}{\alpha+\lambda_{2}-\delta}-\frac{1}{\alpha+\lambda_{2}}\right] \\
& \rightarrow 0\left(\delta \rightarrow 0^{+}\right) .
\end{aligned}
$$

Similarly, we obtain

$$
\begin{aligned}
& \left|k_{\alpha}\left(\lambda_{1}-\delta\right)-k_{\alpha}\left(\lambda_{1}\right)\right| \\
& \quad \leq L_{\alpha}\left[\frac{1}{\alpha+\lambda_{1}-\delta}-\frac{1}{\alpha+\lambda_{1}}+\frac{1}{\alpha+\lambda_{2}}-\frac{1}{\alpha+\lambda_{2}+\delta}\right] \\
& \quad \rightarrow 0\left(\delta \rightarrow 0^{+}\right) .
\end{aligned}
$$

Hence, we derive (22).

\section{Main results and operator expressions}

We also set the following functions:

$$
\begin{aligned}
& \widetilde{\Phi}_{\lambda}(m):=\omega\left(\lambda_{2}, m\right) \frac{\left(\ln U_{m}\right)^{p\left(1-\lambda_{1}\right)-1}}{U_{m}^{1-p} \mu_{m}^{p-1}}, \\
& \widetilde{\Psi}_{\lambda}(n):=\varpi\left(\lambda_{1}, n\right) \frac{\left(\ln V_{n}\right)^{q\left(1-\lambda_{2}\right)-1}}{V_{n}^{1-q} v_{n}^{q-1}} \quad(m, n \in \mathbf{N} \backslash\{1\}) .
\end{aligned}
$$

Theorem 3.1 If $0 \leq-\alpha<\lambda_{1}, \lambda_{2} \leq 1$, then:

(i) for $p>1$, we have the following equivalent inequalities:

$$
\begin{aligned}
I:=\sum_{n=2}^{\infty} \sum_{m=2}^{\infty} \frac{\left(\min \left\{\ln U_{m}, \ln V_{n}\right\}\right)^{\alpha}}{\left(\ln U_{m} V_{n}\right)^{\lambda+\alpha}} a_{m} b_{n} \leq\|a\|_{p, \widetilde{\Phi}_{\lambda}}\|b\|_{q, \widetilde{\Psi}_{\lambda},} \\
J:=\left\{\sum_{n=2}^{\infty} \frac{v_{n} \ln ^{p \lambda_{2}-1} V_{n}}{\left(\varpi\left(\lambda_{1}, n\right)\right)^{p-1} V_{n}}\left[\sum_{m=2}^{\infty} \frac{\left(\min \left\{\ln U_{m}, \ln V_{n}\right\}\right)^{\alpha}}{\left(\ln U_{m} V_{n}\right)^{\lambda+\alpha}} a_{m}\right]^{p}\right\}^{\frac{1}{p}} \\
\leq\|a\|_{p, \widetilde{\Phi}_{\lambda} ;}
\end{aligned}
$$

(ii) for $0<p<1$ (or $p<0)$, we have the equivalent reverses of (24) and (25).

Proof (i) By Hölder's inequality with weight (cf. [11]) and (12), we have 


$$
\begin{aligned}
{\left[\sum_{m=2}^{\infty}\right.} & \left.\frac{\left(\min \left\{\ln U_{m}, \ln V_{n}\right\}\right)^{\alpha}}{\left(\ln U_{m} V_{n}\right)^{\lambda+\alpha}} a_{m}\right]^{p} \\
= & {\left[\sum_{m=2}^{\infty} \frac{\left(\min \left\{\ln U_{m}, \ln V_{n}\right\}\right)^{\alpha}}{\left(\ln U_{m} V_{n}\right)^{\lambda+\alpha}}\right.} \\
& \left.\times\left(\frac{U_{m}^{1 / q}\left(\ln U_{m}\right)^{\left(1-\lambda_{1}\right) / q}}{\left(\ln V_{n}\right)^{\left(1-\lambda_{2}\right) / p} \mu_{m}^{1 / q}} a_{m}\right)\left(\frac{\left(\ln V_{n}\right)^{\left(1-\lambda_{2}\right) / p} \mu_{m}^{1 / q}}{U_{m}^{1 / q}\left(\ln U_{m}\right)^{\left(1-\lambda_{1}\right) / q}}\right)\right]^{p} \\
\leq & \sum_{m=2}^{\infty} \frac{\left(\min \left\{\ln U_{m}, \ln V_{n}\right\}\right)^{\alpha}}{\left(\ln U_{m} V_{n}\right)^{\lambda+\alpha}} \frac{U_{m}^{p-1}\left(\ln U_{m}\right)^{\left(1-\lambda_{1}\right) p / q}}{\left(\ln V_{n}\right)^{1-\lambda_{2}} \mu_{m}^{p / q}} a_{m}^{p} \\
& \times\left[\sum_{m=2}^{\infty} \frac{\left(\min \left\{\ln U_{m}, \ln V_{n}\right\}\right)^{\alpha}}{\left(\ln U_{m} V_{n}\right)^{\lambda+\alpha}} \frac{\left.\left(\ln V_{n}\right)^{\left(1-\lambda_{2}\right)(q-1)} \mu_{m}\right]^{p-1}}{U_{m}\left(\ln U_{m}\right)^{1-\lambda_{1}}}\right] \\
= & \frac{\left(\varpi\left(\lambda_{1}, n\right)\right)^{p-1} V_{n}}{\left(\ln V_{n}\right)^{p \lambda_{2}-1} v_{n}} \\
& \times \sum_{m=2}^{\infty} \frac{\left(\min \left\{\ln U_{m}, \ln V_{n}\right\}\right)^{\alpha}}{\left(\ln U_{m} V_{n}\right)^{\lambda+\alpha}} \frac{v_{n} U_{m}^{p-1}\left(\ln U_{m}\right)^{\left(1-\lambda_{1}\right)(p-1)} a_{m}^{p}}{V_{n}\left(\ln V_{n}\right)^{1-\lambda_{2}} \mu_{m}^{p-1}} .
\end{aligned}
$$

Hence by (11), we deduce that

$$
\begin{aligned}
J & \leq\left[\sum_{n=2}^{\infty} \sum_{m=2}^{\infty} \frac{\left(\min \left\{\ln U_{m}, \ln V_{n}\right\}\right)^{\alpha}}{\left(\ln U_{m} V_{n}\right)^{\lambda+\alpha}} \frac{v_{n} U_{m}^{p-1}\left(\ln U_{m}\right)^{\left(1-\lambda_{1}\right)(p-1)}}{V_{n}\left(\ln V_{n}\right)^{1-\lambda_{2}} \mu_{m}^{p-1}} a_{m}^{p}\right]^{\frac{1}{p}} \\
& =\left[\sum_{m=2}^{\infty} \sum_{n=2}^{\infty} \frac{\left(\min \left\{\ln U_{m}, \ln V_{n}\right\}\right)^{\alpha}}{\left(\ln U_{m} V_{n}\right)^{\lambda+\alpha}} \frac{v_{n}\left(\ln U_{m}\right)^{\lambda_{1}}}{V_{n}\left(\ln V_{n}\right)^{1-\lambda_{2}}} \frac{\left(\ln U_{m}\right)^{p\left(1-\lambda_{1}\right)-1}}{U_{m}^{1-p} \mu_{m}^{p-1}} a_{m}^{p}\right]^{\frac{1}{p}} \\
& =\left[\sum_{m=2}^{\infty} \omega\left(\lambda_{2}, m\right) \frac{\left(\ln U_{m}\right)^{p\left(1-\lambda_{1}\right)-1}}{U_{m}^{1-p} \mu_{m}^{p-1}} a_{m}^{p}\right]^{\frac{1}{p}},
\end{aligned}
$$

and then (25) follows.

By Hölder's inequality (cf. [11]), we have

$$
\begin{aligned}
& I=\sum_{n=2}^{\infty}\left[\frac{\left(\ln V_{n}\right)^{\lambda_{2}-\frac{1}{p}} v_{n}^{1 / p}}{\left(\varpi\left(\lambda_{1}, n\right)\right)^{\frac{1}{q}} V_{n}^{\frac{1}{p}}} \sum_{m=2}^{\infty} \frac{\left(\min \left\{\ln U_{m}, \ln V_{n}\right\}\right)^{\alpha}}{\left(\ln U_{m} V_{n}\right)^{\lambda+\alpha}} a_{m}\right] \\
& \times\left[\left(\varpi\left(\lambda_{1}, n\right)\right)^{\frac{1}{q}} \frac{\left(\ln V_{n}\right)^{\frac{1}{p}-\lambda_{2}}}{V_{n}^{\frac{-1}{p}} v_{n}^{\frac{1}{p}}} b_{n}\right] \leq J\|b\|_{q, \widetilde{\Psi}_{i}} .
\end{aligned}
$$

Then by (25), we derive (24).

On the other hand, assuming that (24) holds true, we set

$$
b_{n}:=\frac{\left(\ln V_{n}\right)^{p \lambda_{2}-1} v_{n}}{\left(\varpi\left(\lambda_{1}, n\right)\right)^{p-1} V_{n}}\left[\sum_{m=2}^{\infty} \frac{\left(\min \left\{\ln U_{m}, \ln V_{n}\right\}\right)^{\alpha}}{\left(\ln U_{m} V_{n}\right)^{\lambda+\alpha}} a_{m}\right]^{p-1}, \quad n \in \mathbf{N} \backslash\{1\} .
$$


Then we obtain that $J^{p}=\|b\|_{q, \Psi_{\lambda}}^{q}$. If $J=0$, then (25) is trivially valid; if $J=\infty$, then by (27), (25) takes the form of equality $(=\infty)$. Suppose that $0<J<\infty$. By (24), it follows that

$$
\begin{gathered}
\|b\|_{q, \widetilde{\Psi}_{\lambda}}^{q}=J^{p}=I \leq\|a\|_{p, \widetilde{\Phi}_{\lambda}}\|b\|_{q, \widetilde{\Psi}_{\lambda}}, \\
\|b\|_{q, \widetilde{\Psi}_{\lambda}}^{q-1}=J \leq\|a\|_{p, \widetilde{\Phi}_{\lambda}},
\end{gathered}
$$

and then (25) follows, which is equivalent to (24).

(ii) For $0<p<1$ (or $p<0$ ), by the reverse Hölder inequality with weight (cf. [11]) and (12), we obtain the reverse of (26) (or (26)). Then by (11), we derive the reverse of (27), and thus the reverse of (25) follows. By Hölder's inequality (cf. [11]), we obtain the reverse of (28) and then by the reverse of (25), we deduce the reverse of (24).

On the other hand, assuming that the reverse of (24) holds true, we set $b_{n}$ as in (29). Then we obtain that $J^{p}=\|b\|_{q, \widetilde{\Psi}_{i}}^{q}$. If $J=\infty$, then the reverse of (25) is trivially valid; if $J=0$, then by the reverse of (27), (25) takes the form of equality (=0). Suppose that $0<J<\infty$. By the reverse of (24), it follows that the reverses of (30) and (31) are valid and then the reverse of (25) follows, which is equivalent to the reverse of (24).

Theorem 3.2 If $0 \leq-\alpha<\lambda_{1}, \lambda_{2} \leq 1, k_{\alpha}\left(\lambda_{1}\right)$ is defined as in (8), there exist $m_{0}, n_{0} \in \mathbf{N}$, such that $\left\{\mu_{m}\right\}_{m=m_{0}}^{\infty}$ and $\left\{v_{n}\right\}_{n=n_{0}}^{\infty}$ are decreasing, $U_{\infty}=V_{\infty}=\infty$, then for $p>1,\|a\|_{p, \Phi_{\lambda}} \in \mathbf{R}_{+}$and $\|b\|_{q, \Psi_{\lambda}} \in \mathbf{R}_{+}$, we have the following equivalent inequalities:

$$
\begin{aligned}
\sum_{n=2}^{\infty} & \sum_{m=2}^{\infty} \frac{\left(\min \left\{\ln U_{m}, \ln V_{n}\right\}\right)^{\alpha}}{\left(\ln U_{m} V_{n}\right)^{\lambda+\alpha}} a_{m} b_{n}<k_{\alpha}\left(\lambda_{1}\right)\|a\|_{p, \Phi_{\lambda}}\|b\|_{q, \Psi_{\lambda}} \\
J_{1} & :=\left\{\sum_{n=2}^{\infty} \frac{v_{n} \ln ^{p \lambda_{2}-1} V_{n}}{V_{n}}\left[\sum_{m=2}^{\infty} \frac{\left(\min \left\{\ln U_{m}, \ln V_{n}\right\}\right)^{\alpha}}{\left(\ln U_{m} V_{n}\right)^{\lambda+\alpha}} a_{m}\right]^{p}\right\}^{\frac{1}{p}} \\
& <k_{\alpha}\left(\lambda_{1}\right)\|a\|_{p, \Phi_{\lambda}},
\end{aligned}
$$

where the constant factor $k_{\alpha}\left(\lambda_{1}\right)$ is the best possible.

Proof Applying (13) and (14) in (24) and (25), since

$$
\left(\omega\left(\lambda_{2}, m\right)\right)^{\frac{1}{p}}<\left(k_{\alpha}\left(\lambda_{1}\right)\right)^{\frac{1}{p}} \quad(p>1), \quad\left(\varpi\left(\lambda_{1}, n\right)\right)^{\frac{1}{q}}<\left(k_{\alpha}\left(\lambda_{1}\right)\right)^{\frac{1}{q}} \quad(q>1),
$$

and

$$
\frac{1}{\left(k_{\alpha}\left(\lambda_{1}\right)\right)^{p-1}}<\frac{1}{\left(\varpi\left(\lambda_{1}, n\right)\right)^{p-1}} \quad(p>1),
$$

by simplification, we obtain the equivalent inequalities (32) and (33). 
For $\varepsilon \in\left(0, q\left(\alpha+\lambda_{2}\right)\right)$, we set $\widetilde{\lambda}_{1}=\lambda_{1}+\frac{\varepsilon}{q}(>-\alpha), \tilde{\lambda}_{2}=\lambda_{2}-\frac{\varepsilon}{q}(\in(-\alpha, 1))$, and $\widetilde{a}=\left\{\widetilde{a}_{m}\right\}_{m=2}^{\infty}, \widetilde{b}=\left\{\widetilde{b}_{n}\right\}_{n=2}^{\infty}$,

$$
\begin{aligned}
\widetilde{a}_{m} & :=\frac{\mu_{m}}{U_{m}} \ln ^{\tilde{\lambda}_{1}-\varepsilon-1} U_{m}=\frac{\mu_{m}}{U_{m}} \ln ^{\lambda_{1}-\frac{\varepsilon}{p}-1} U_{m}, \\
\widetilde{b}_{n} & =\frac{v_{n}}{V_{n}} \ln ^{\tilde{\lambda}_{2}-1} V_{n}=\frac{v_{n}}{V_{n}} \ln ^{\lambda_{2}-\frac{\varepsilon}{q}-1} V_{n} .
\end{aligned}
$$

Then by (20), (21) and (19), we have

$$
\begin{aligned}
&\|\widetilde{a}\|_{p, \Phi_{\lambda}}\|\widetilde{b}\|_{q, \Psi_{\lambda}}=\left(\sum_{m=2}^{\infty} \frac{\mu_{m}}{U_{m} \ln ^{1+\varepsilon} U_{m}}\right)^{\frac{1}{p}}\left(\sum_{n=2}^{\infty} \frac{v_{n}}{V_{n} \ln ^{1+\varepsilon} V_{n}}\right)^{\frac{1}{q}} \\
&=\frac{1}{\varepsilon}\left[\frac{1}{\ln ^{\varepsilon} U_{m_{0}+1}}+\varepsilon O_{1}(1)\right]^{\frac{1}{p}}\left[\frac{1}{\ln ^{\varepsilon} V_{n_{0}+1}}+\varepsilon O_{2}(1)\right]^{\frac{1}{q}}, \\
& \widetilde{I}:=\sum_{n=2}^{\infty} \sum_{m=2}^{\infty} \frac{\left(\min \left\{\ln U_{m}, \ln V_{n}\right\}\right)^{\alpha}}{\left(\ln U_{m} V_{n}\right)^{\lambda+\alpha}} \widetilde{a}_{m} \widetilde{b}_{n} \\
&=\sum_{m=2}^{\infty}\left[\sum_{n=2}^{\infty} \frac{\left(\min \left\{\ln U_{m}, \ln V_{n}\right\}\right)^{\alpha}}{\left(\ln U_{m} V_{n}\right)^{\lambda+\alpha}} \frac{v_{n} \ln ^{\hat{\lambda}_{1}} U_{m}}{V_{n} \ln ^{1-\tilde{\lambda}_{2}} V_{n}}\right] \frac{\mu_{m}}{U_{m} \ln ^{\varepsilon+1} U_{m}} \\
&=\sum_{m=2}^{\infty} \omega\left(\tilde{\lambda}_{2}, m\right) \frac{\mu_{m}}{U_{m} \ln ^{\varepsilon+1} U_{m}} \\
& \geq k_{\alpha}\left(\tilde{\lambda}_{1}\right) \sum_{m=2}^{\infty}\left(1-O\left(\frac{1}{\ln ^{\lambda_{2}+\alpha} U_{m}}\right)\right) \frac{\mu_{m}}{U_{m} \ln \varepsilon+1} U_{m} \\
&=k_{\alpha}\left(\widetilde{\lambda}_{1}\right)\left[\sum_{m=2}^{\infty} \frac{\mu_{m}}{U_{m} \ln ^{\varepsilon+1} U_{m}}-\sum_{m=2}^{\infty} O\left(\frac{\mu_{m}}{U_{m}\left(\ln U_{m}\right)^{\left(\frac{\varepsilon}{p}+\alpha+\lambda_{2}\right)+1}}\right)\right] \\
&=\frac{1}{\varepsilon} k_{\alpha}\left(\lambda_{1}+\frac{\varepsilon}{q}\right)\left[\frac{1}{\ln ^{\varepsilon} U_{m}+1}+\varepsilon\left(O_{1}(1)-O(1)\right)\right] .
\end{aligned}
$$

If there exists a positive constant $K \leq k_{\alpha}\left(\lambda_{1}\right)$, such that (32) is valid when replacing $k_{\alpha}\left(\lambda_{1}\right)$ by $K$, then in particular, we have $\varepsilon \widetilde{I}<\varepsilon K\|\widetilde{a}\|_{p, \Phi_{\lambda}}\|\widetilde{b}\|_{q, \Psi_{\lambda}}$, namely

$$
\begin{aligned}
& k_{\alpha}\left(\lambda_{1}+\frac{\varepsilon}{q}\right)\left[\frac{1}{\ln ^{\varepsilon} U_{m_{0}+1}}+\varepsilon\left(O_{1}(1)-O(1)\right)\right] \\
& \quad<K\left[\frac{1}{\ln ^{\varepsilon} U_{m_{0}+1}}+\varepsilon O_{1}(1)\right]^{\frac{1}{p}}\left[\frac{1}{\ln ^{\varepsilon} V_{n_{0}+1}}+\varepsilon O_{2}(1)\right]^{\frac{1}{q}} .
\end{aligned}
$$

In view of (22), it follows that $k_{\alpha}\left(\lambda_{1}\right) \leq K\left(\varepsilon \rightarrow 0^{+}\right)$. Hence, $K=k_{\alpha}\left(\lambda_{1}\right)$ is the best possible constant factor of (32).

Similarly to (28), we can still obtain that

$$
I \leq J_{1}\|b\|_{q, \Psi_{\lambda}}
$$


Hence, we can prove that the constant factor $k_{\alpha}\left(\lambda_{1}\right)$ in (33) is the best possible. Otherwise, we would reach a contradiction by (36) that the constant factor in (32) is not the best possible.

For $p>1$,

$$
\Psi_{\lambda}^{1-p}(n)=\frac{v_{n}}{V_{n}}\left(\ln V_{n}\right)^{p \lambda_{2}-1} \quad(n \in \mathbf{N} \backslash\{1\}),
$$

we define the following normed spaces:

$$
\begin{aligned}
l_{p, \Phi_{\lambda}}: & =\left\{a=\left\{a_{m}\right\}_{m=2}^{\infty} ;\|a\|_{p, \Phi_{\lambda}}<\infty\right\}, \\
l_{q, \Psi_{\lambda}} & :=\left\{b=\left\{b_{n}\right\}_{n=2}^{\infty} ;\|b\|_{q, \Psi_{\lambda}}<\infty\right\}, \\
l_{p, \Psi_{\lambda}^{1-p}}: & =\left\{c=\left\{c_{n}\right\}_{n=2}^{\infty} ;\|c\|_{p, \Psi_{\lambda}^{1-p}}<\infty\right\} .
\end{aligned}
$$

Assuming that $a=\left\{a_{m}\right\}_{m=2}^{\infty} \in l_{p, \Phi_{\lambda}}$, setting

$$
c=\left\{c_{n}\right\}_{n=2}^{\infty}, \quad c_{n}:=\sum_{m=2}^{\infty} \frac{\left(\min \left\{\ln U_{m}, \ln V_{n}\right\}\right)^{\alpha}}{\left(\ln U_{m} V_{n}\right)^{\lambda+\alpha}} a_{m}, n \in \mathbf{N} \backslash\{1\},
$$

we can rewrite (33) as follows:

$$
\|c\|_{p, \Psi_{\lambda}^{1-p}}<k_{\alpha}\left(\lambda_{1}\right)\|a\|_{p, \Phi_{\lambda}}<\infty
$$

namely, $c \in l_{p, \Psi_{\lambda}^{1-p}}$.

Definition 3.3 Define a Hardy-Mulholland-type operator $T: l_{p, \Phi_{\lambda}} \rightarrow l_{p, \Psi_{\lambda}^{1-p}}$ as follows: For any $a=\left\{a_{m}\right\}_{m=2}^{\infty} \in l_{p, \Phi_{\lambda}}$, there exists a unique representation $T a=c \in l_{p, \Psi_{\lambda}^{1-p}}$. Define the formal inner product of $T a$ and $b=\left\{b_{n}\right\}_{n=2}^{\infty} \in l_{q, \Psi_{\lambda}}$ as follows:

$$
(T a, b):=\sum_{n=2}^{\infty}\left[\sum_{m=2}^{\infty} \frac{\left(\min \left\{\ln U_{m}, \ln V_{n}\right\}\right)^{\alpha}}{\left(\ln U_{m} V_{n}\right)^{\lambda+\alpha}} a_{m}\right] b_{n}
$$

Then we can rewrite (32) and (33) as bellow:

$$
\begin{gathered}
(T a, b)<k_{\alpha}\left(\lambda_{1}\right)\|a\|_{p, \Phi_{\lambda}}\|b\|_{q, \Psi_{\lambda}}, \\
\|T a\|_{p, \Psi_{\lambda}^{1-p}}<k_{\alpha}\left(\lambda_{1}\right)\|a\|_{p, \Phi_{\lambda}} .
\end{gathered}
$$

Define the norm of operator $T$ as follows: 


$$
\|T\|:=\sup _{a(\neq \theta) \in l_{p, \Phi_{\lambda}}} \frac{\|T a\|_{p, \Psi_{\lambda}^{1-p}}}{\|a\|_{p, \Phi_{\lambda}}} .
$$

Then by (39), we derive that $\|T\| \leq k_{\alpha}\left(\lambda_{1}\right)$. Since the constant factor in (39) is the best possible, we have

$$
\|T\|=k_{\alpha}\left(\lambda_{1}\right)=\sum_{i=1}^{2} \frac{1}{\lambda_{i}+\alpha} F\left(\lambda+\alpha, \lambda_{i}+\alpha, \lambda_{i}+\alpha+1,-1\right) .
$$

\section{Some equivalent reverses}

In the following, we also set

$$
\begin{aligned}
& \widetilde{\Omega}_{\lambda}(m):=\left(1-\theta\left(\lambda_{2}, m\right)\right) \frac{\left(\ln U_{m}\right)^{p\left(1-\lambda_{1}\right)-1}}{U_{m}^{1-p} \mu_{m}^{p-1}}, \\
& \widetilde{\Upsilon}_{\lambda}(n):=\left(1-\vartheta\left(\lambda_{1}, n\right)\right) \frac{\left(\ln V_{n}\right)^{q\left(1-\lambda_{2}\right)-1}}{V_{n}^{1-q} v_{n}^{q-1}} \quad(m, n \in \mathbf{N} \backslash\{1\}) .
\end{aligned}
$$

For $0<p<1$ or $p<0$, we still use $\|a\|_{p, \Phi_{\lambda}},\|b\|_{q, \Psi_{\lambda}},\|a\|_{p, \widetilde{\Omega}_{\lambda}}$ and $\|b\|_{q, \widetilde{\Upsilon}_{\lambda}}$ as the formal symbols.

Theorem 4.1 With regard to the assumptions of Theorem 3.2, if $0<p<1$, $\|a\|_{p, \Phi_{\lambda}} \in \mathbf{R}_{+}$and $\|b\|_{q, \Psi_{\lambda}} \in \mathbf{R}_{+}$, then we have the following equivalent inequalities with the best possible constant factor $k_{\alpha}\left(\lambda_{1}\right)$ :

$$
\begin{gathered}
\sum_{n=2}^{\infty} \sum_{m=2}^{\infty} \frac{\left(\min \left\{\ln U_{m}, \ln V_{n}\right\}\right)^{\alpha}}{\left(\ln U_{m} V_{n}\right)^{\lambda+\alpha}} a_{m} b_{n}>k_{\alpha}\left(\lambda_{1}\right)\|a\|_{p, \widetilde{\Omega}_{\lambda}}\|b\|_{q, \Psi_{\lambda},} \\
\left\{\sum_{n=2}^{\infty} \frac{v_{n} \ln ^{p \lambda_{2}-1} V_{n}}{V_{n}}\left[\sum_{m=2}^{\infty} \frac{\left(\min \left\{\ln U_{m}, \ln V_{n}\right\}\right)^{\alpha}}{\left(\ln U_{m} V_{n}\right)^{\lambda+\alpha}} a_{m}\right]^{p}\right\}^{\frac{1}{p}}>k_{\alpha}\left(\lambda_{1}\right)\|a\|_{p, \widetilde{\Omega}_{\lambda}}
\end{gathered}
$$

Proof Using (16) and (14) in the reverses of (24) and (25), since

$$
\begin{aligned}
& \left(\omega\left(\lambda_{2}, m\right)\right)^{\frac{1}{p}}>\left(k_{\alpha}\left(\lambda_{1}\right)\right)^{\frac{1}{p}}\left(1-\theta\left(\lambda_{2}, m\right)\right)^{\frac{1}{p}} \quad(0<p<1), \\
& \left(\varpi\left(\lambda_{1}, n\right)\right)^{\frac{1}{q}}>\left(k_{\alpha}\left(\lambda_{1}\right)\right)^{\frac{1}{q}} \quad(q<0),
\end{aligned}
$$

and 


$$
\frac{1}{\left(k_{\alpha}\left(\lambda_{1}\right)\right)^{p-1}}>\frac{1}{\left(\varpi\left(\lambda_{1}, n\right)\right)^{p-1}} \quad(0<p<1)
$$

by simplification, we obtain the equivalent inequalities (42) and (43).

For $\varepsilon \in\left(0, p\left(\alpha+\lambda_{1}\right)\right)$, we set

$$
\begin{aligned}
& \widetilde{\lambda}_{1}=\lambda_{1}-\frac{\varepsilon}{p}(\in(-\alpha, 1)), \quad \widetilde{\lambda}_{2}=\lambda_{2}+\frac{\varepsilon}{p}(>-\alpha), \quad \text { and } \\
& \widetilde{a}=\left\{\widetilde{a}_{m}\right\}_{m=2}^{\infty}, \quad \widetilde{b}=\left\{\widetilde{b}_{n}\right\}_{n=2}^{\infty}, \\
& \widetilde{a}_{m}:=\frac{\mu_{m}}{U_{m}} \ln ^{\tilde{\lambda}_{1}-1} U_{m}=\frac{\mu_{m}}{U_{m}} \ln ^{\lambda_{1}-\frac{\varepsilon}{p}-1} U_{m}, \\
& \widetilde{b}_{n}=\frac{v_{n}}{V_{n}} \ln ^{\widetilde{\lambda}_{2}-\varepsilon-1} V_{n}=\frac{v_{n}}{V_{n}} \ln ^{\lambda_{2}-\frac{\varepsilon}{q}-1} V_{n} .
\end{aligned}
$$

Then by (20), (21) and (14), we obtain that

$$
\begin{aligned}
\|a\|_{p, \widetilde{\Omega}_{\lambda}} & \|b\|_{q, \Psi_{\lambda}} \\
= & {\left[\sum_{m=2}^{\infty}\left(1-\theta\left(\lambda_{2}, m\right)\right) \frac{\mu_{m}}{U_{m} \ln ^{1+\varepsilon} U_{m}}\right]^{\frac{1}{p}}\left(\sum_{n=2}^{\infty} \frac{v_{n}}{V_{n} \ln ^{1+\varepsilon} V_{n}}\right)^{\frac{1}{q}} } \\
= & \left(\sum_{m=2}^{\infty} \frac{\mu_{m}}{U_{m} \ln ^{1+\varepsilon} U_{m}}-\sum_{m=2}^{\infty} O\left(\frac{\mu_{m}}{U_{m} \ln ^{1+\alpha+\lambda_{2}+\varepsilon} U_{m}}\right)\right)^{\frac{1}{p}} \\
& \times\left(\sum_{n=2}^{\infty} \frac{v_{n}}{V_{n} \ln ^{1+\varepsilon} V_{n}}\right)^{\frac{1}{q}} \\
= & \frac{1}{\varepsilon}\left[\frac{1}{\ln U_{m m_{0}+1}}+\varepsilon\left(O_{1}(1)-O(1)\right)\right]^{\frac{1}{p}}\left[\frac{1}{\ln ^{\varepsilon} V_{n_{0}+1}}+\varepsilon O_{2}(1)\right]^{\frac{1}{q}}, \\
\widetilde{I}:= & \sum_{n=2}^{\infty} \sum_{m=2}^{\infty} \frac{\left(\min \left\{\ln U_{m}, \ln V_{n}\right\}\right)^{\alpha}}{\left(\ln U_{m} V_{n}\right)^{\lambda+\alpha}} \widetilde{a}_{m} \widetilde{b}_{n} \\
= & \sum_{n=2}^{\infty}\left[\sum_{m=2}^{\infty} \frac{\left(\min \left\{\ln U_{m}, \ln V_{n}\right\}\right)^{\alpha}}{\left(\ln U_{m} V_{n}\right)^{\lambda+\alpha}} \frac{\mu_{m} \ln ^{\lambda_{2}} V_{n}}{U_{m} \ln ^{1-\tilde{\lambda}_{1}} U_{m}}\right] \frac{v_{n}}{V_{n} \ln ^{\varepsilon+1} V_{n}} \\
= & \sum_{n=2}^{\infty} \varpi\left(\widetilde{\lambda}_{1}, n\right) \frac{v_{n}}{V_{n} \ln ^{\varepsilon+1} V_{n}} \leq k_{\alpha}\left(\widetilde{\lambda}_{1}\right) \sum_{n=2}^{\infty} \frac{v_{n}}{V_{n} \ln ^{\varepsilon+1} V_{n}} \\
= & \frac{1}{\varepsilon} k_{\alpha}\left(\lambda_{1}-\frac{\varepsilon}{p}\right)\left[\frac{1}{\ln ^{\varepsilon} V_{n_{0}+1}}+\varepsilon O_{2}(1)\right] .
\end{aligned}
$$

If there exists a positive constant $K \geq k_{\alpha}\left(\lambda_{1}\right)$, such that (42) is valid when replacing $k_{\alpha}\left(\lambda_{1}\right)$ by $K$, then in particular, we have $\varepsilon \widetilde{I}>\varepsilon K\|\widetilde{a}\|_{p, \Omega_{\lambda}}\|\widetilde{b}\|_{q, \Psi_{\lambda}}$, namely 


$$
\begin{aligned}
& k_{\alpha}\left(\lambda_{1}-\frac{\varepsilon}{p}\right)\left[\frac{1}{\ln ^{\varepsilon} V_{n_{0}+1}}+\varepsilon O_{2}(1)\right] \\
& \quad>K\left[\frac{1}{\ln ^{\varepsilon} U_{m_{0}+1}}+\varepsilon\left(O_{1}(1)-O(1)\right)\right]^{\frac{1}{p}}\left[\frac{1}{\ln ^{\varepsilon} V_{n_{0}+1}}+\varepsilon O_{2}(1)\right]^{\frac{1}{q}} .
\end{aligned}
$$

In view of (22), it follows that $k_{\alpha}\left(\lambda_{1}\right) \geq K\left(\varepsilon \rightarrow 0^{+}\right)$. Hence, $K=k_{\alpha}\left(\lambda_{1}\right)$ is the best possible constant factor of (42). The constant factor $k_{\alpha}\left(\lambda_{1}\right)$ in (43) is still the best possible. Otherwise, we would reach a contradiction by the reverse of (36) that the constant factor in (42) is not the best possible.

Theorem 4.2 With regard to the assumptions of Theorem 3.2, if $p<0,\|a\|_{p, \Phi_{\lambda}} \in$ $\mathbf{R}_{+}$and $\|b\|_{q, \Psi_{\lambda}} \in \mathbf{R}_{+}$, then we have the following equivalent inequalities with the best possible constant factor $B\left(\lambda_{1}, \lambda_{2}\right)$ :

$$
\begin{aligned}
& \sum_{n=2}^{\infty} \sum_{m=2}^{\infty} \frac{\left(\min \left\{\ln U_{m}, \ln V_{n}\right\}\right)^{\alpha}}{\left(\ln U_{m} V_{n}\right)^{\lambda+\alpha}} a_{m} b_{n}>k_{\alpha}\left(\lambda_{1}\right)\|a\|_{p, \Phi_{i}}\|b\|_{q, \widetilde{\Upsilon}_{\lambda}}, \\
J_{2}:= & \left\{\sum_{n=2}^{\infty} \frac{v_{n} \ln ^{p \lambda_{2}-1} V_{n}}{\left(1-\vartheta\left(\lambda_{1}, n\right)\right)^{p-1} V_{n}}\left[\sum_{m=2}^{\infty} \frac{\left(\min \left\{\ln U_{m}, \ln V_{n}\right\}\right)^{\alpha}}{\left(\ln U_{m} V_{n}\right)^{\lambda+\alpha}} a_{m}\right]^{p}\right\}^{\frac{1}{p}} \\
> & k_{\alpha}\left(\lambda_{1}\right)\|a\|_{p, \Phi_{\lambda}} .
\end{aligned}
$$

Proof Using (13) and (17) in the reverses of (24) and (25), since

$$
\begin{aligned}
& \left(\omega\left(\lambda_{2}, m\right)\right)^{\frac{1}{p}}>\left(k_{\alpha}\left(\lambda_{1}\right)\right)^{\frac{1}{p}} \quad(p<0), \\
& \left(\varpi\left(\lambda_{1}, n\right)\right)^{\frac{1}{q}}>\left(k_{\alpha}\left(\lambda_{1}\right)\right)^{\frac{1}{q}}\left(1-\vartheta\left(\lambda_{1}, n\right)\right)^{\frac{1}{q}} \quad(0<q<1),
\end{aligned}
$$

and

$$
\left[\frac{1}{\left(k_{\alpha}\left(\lambda_{1}\right)\right)^{p-1}\left(1-\vartheta\left(\lambda_{1}, n\right)\right)^{p-1}}\right]^{\frac{1}{p}}>\left[\frac{1}{\left(\varpi\left(\lambda_{1}, n\right)\right)^{p-1}}\right]^{\frac{1}{p}} \quad(p<0),
$$

by simplification, we obtain equivalent inequalities (44) and (45).

For $\varepsilon \in\left(0, q\left(\alpha+\lambda_{2}\right)\right)$, we set

$$
\begin{aligned}
& \tilde{\lambda}_{1}=\lambda_{1}+\frac{\varepsilon}{q}(>-\alpha), \quad \tilde{\lambda}_{2}=\lambda_{2}-\frac{\varepsilon}{q}(\in(-\alpha, 1)), \quad \text { and } \quad \widetilde{a}=\left\{\widetilde{a}_{m}\right\}_{m=2}^{\infty}, \quad \tilde{b}=\left\{\widetilde{b}_{n}\right\}_{n=2}^{\infty}, \\
& \widetilde{a}_{m}=\frac{\mu_{m}}{U_{m}} \ln ^{\tilde{\lambda}_{1}-\varepsilon-1} U_{m}=\frac{\mu_{m}}{U_{m}} \ln ^{\lambda_{1}-\frac{\varepsilon}{p}-1} U_{m}, \\
& \widetilde{b}_{n}=\frac{v_{n}}{V_{n}} \ln ^{\tilde{\lambda}_{2}-1} V_{n}=\frac{v_{n}}{V_{n}} \ln ^{\lambda_{2}-\frac{\varepsilon}{q}-1} V_{n} .
\end{aligned}
$$

Then by (20), (21) and (12), we have 


$$
\begin{aligned}
\|\widetilde{a}\|_{p, \Phi_{\lambda}}\|\widetilde{b}\|_{q, \tilde{q}_{\lambda}} & \left(\sum_{m=2}^{\infty} \frac{\mu_{m}}{U_{m} \ln ^{\varepsilon+1} U_{m}}\right)^{\frac{1}{p}}\left[\sum_{n=2}^{\infty}\left(1-\vartheta\left(\lambda_{1}, n\right)\right) \frac{v_{n}}{V_{n} \ln ^{\varepsilon+1} V_{n}}\right]^{\frac{1}{q}} \\
= & \left(\sum_{m=2}^{\infty} \frac{\mu_{m}}{U_{m} \ln ^{\varepsilon+1} U_{m}}\right)^{\frac{1}{p}} \\
& \times\left[\sum_{n=2}^{\infty} \frac{v_{n}}{V_{n} \ln ^{\varepsilon+1} V_{n}}-\sum_{n=2}^{\infty} O\left(\frac{v_{n}}{V_{n} \ln ^{1+\left(\alpha+\lambda_{1}+\varepsilon\right)} V_{n}}\right)\right]^{\frac{1}{q}} \\
= & \frac{1}{\varepsilon}\left[\frac{1}{\ln \varepsilon} U_{m_{0}+1}+\varepsilon O_{1}(1)\right]^{\frac{1}{p}}\left[\frac{1}{\ln ^{\varepsilon} V_{n_{0}+1}}+\varepsilon\left(O_{2}(1)-O(1)\right)\right]^{\frac{1}{q}}, \\
\widetilde{I}= & \sum_{m=2}^{\infty} \sum_{n=2}^{\infty} \frac{\left(\min \left\{\ln U_{m}, \ln _{n}\right\}\right)^{\alpha}}{\left(\ln U_{m} V_{n}\right)^{\lambda+\alpha}} \widetilde{a}_{m} \widetilde{b}_{n} \\
= & \sum_{m=2}^{\infty}\left[\sum_{n=2}^{\infty} \frac{\tilde{l n}^{\lambda_{1}} U_{m}}{\ln ^{\lambda}\left(U_{m} V_{n}\right)} \frac{v_{n}}{V_{n}} \tilde{l n}^{\hat{\lambda}_{2}-1} V_{n}\right] \frac{\mu_{m}}{U_{m} \ln ^{\varepsilon+1} U_{m}} \\
= & \sum_{m=2}^{\infty} \omega\left(\widetilde{\lambda}_{2}, m\right) \frac{\mu_{m}}{U_{m} \ln ^{\varepsilon+1} U_{m}} \leq k_{\alpha}\left(\widetilde{\lambda}_{1}\right) \sum_{n=2}^{\infty} \frac{\mu_{m}}{U_{m} \ln ^{\varepsilon+1} U_{m}} \\
= & \frac{1}{\varepsilon} k_{\alpha}\left(\lambda_{1}+\frac{\varepsilon}{q}\right)\left[\frac{1}{\ln ^{\varepsilon} U_{m}+1}+\varepsilon O_{1}(1)\right] .
\end{aligned}
$$

If there exists a positive constant $K \geq k_{\alpha}\left(\lambda_{1}\right)$, such that (44) is satisfied when replacing $k_{\alpha}\left(\lambda_{1}\right)$ by $K$, then in particular, we have $\varepsilon \widetilde{I}>\varepsilon K\|\widetilde{a}\|_{p, \Phi_{\lambda}}\|\widetilde{b}\|_{q, \widetilde{\Upsilon}_{\lambda}}$, namely

$$
\begin{aligned}
& k_{\alpha}\left(\lambda_{1}+\frac{\varepsilon}{q}\right)\left[\frac{1}{\ln ^{\varepsilon} U_{m_{0}+1}}+\varepsilon O_{1}(1)\right]>K\left[\frac{1}{\ln ^{\varepsilon} U_{m_{0}+1}}+\varepsilon O_{1}(1)\right]^{\frac{1}{p}} \\
& \quad \times\left[\frac{1}{\ln ^{\varepsilon} V_{n_{0}+1}}+\varepsilon\left(O_{2}(1)-O(1)\right)\right]^{\frac{1}{q}} .
\end{aligned}
$$

In view of (22), it follows that $k_{\alpha}\left(\lambda_{1}\right) \geq K\left(\varepsilon \rightarrow 0^{+}\right)$. Hence, $K=k_{\alpha}\left(\lambda_{1}\right)$ is the best possible constant factor of (44). Similarly to the reverse of (28), we still obtain that

$$
I \geq J_{2}\|b\|_{q, \widetilde{\Upsilon}_{\lambda}}
$$

Hence, the constant factor $k_{\alpha}\left(\lambda_{1}\right)$ in (45) is still the best possible. Otherwise, we would reach a contradiction by (46) that the constant factor in (44) is not the best possible.

Remark 4.3 For $\alpha=0$ in (32), by (9), we have 


$$
\sum_{n=2}^{\infty} \sum_{m=2}^{\infty} \frac{a_{m} b_{n}}{\left(\ln U_{m} V_{n}\right)^{\lambda}}<B\left(\lambda_{1}, \lambda_{2}\right)\|a\|_{p, \Phi_{\lambda}}\|b\|_{q, \Psi_{\lambda}} .
$$

For $\lambda=1, \lambda_{1}=\frac{1}{q}, \lambda_{2}=\frac{1}{p}, \mu_{i}=v_{i}=1(i \in \mathbf{N})$ in (47), we deduce (2). Hence, (47) is an extension of (2); so is (32).

Acknowledgements We are thankful to the mathematicians who have read the manuscript of the paper, for their constructive comments that helped improve its presentation.

Author Contributions The authors have contributed equally for the preparation of the present paper.

Funding Open Access funding provided by University of Zurich. B. Yang: This work is supported by the National Natural Science Foundation (no. 61772140), and Science and Technology Planning Project Item of Guangzhou City (no. 201707010229). We are grateful for their support. A. M. Raigorodskii: The research was partially supported by the grant NSh-2540.2020.1 of the Russian President supporting leading scientific schools of Russia

Availability of data and materials Not applicable.

\section{Compliance with ethical standards}

Competing interests The authors of the present paper do not have any competing interests.

Open Access This article is licensed under a Creative Commons Attribution 4.0 International License, which permits use, sharing, adaptation, distribution and reproduction in any medium or format, as long as you give appropriate credit to the original author(s) and the source, provide a link to the Creative Commons licence, and indicate if changes were made. The images or other third party material in this article are included in the article's Creative Commons licence, unless indicated otherwise in a credit line to the material. If material is not included in the article's Creative Commons licence and your intended use is not permitted by statutory regulation or exceeds the permitted use, you will need to obtain permission directly from the copyright holder. To view a copy of this licence, visit http:// creativecommons.org/licenses/by/4.0/.

\section{References}

1. Arpad, B., Choonghong, O.: Best constant for certain multilinear integral operator. J. Inequal. Appl. 28582 (2006). https://doi.org/10.1155/JIA/2006/28582

2. Azar, L.: On some extensions of Hardy-Hilbert's inequality and applications. J. Inequal. Appl. 546829 (2009). https://doi.org/10.1155/2008/546829

3. Chen, Q., He, B., Hong, Y., Zhen, L.: Equivalent parameter conditions for the validity of half-discrete Hilbert-type multiple integral inequality with generalized homogeneous kernel. J. Funct. Spaces. 2020 (2020). Article ID 7414861

4. Gao, P.: On weight Hardy inequalities for non-increasing sequence. J. Math. Inequal. 12(2), 551-557 (2018)

5. Hardy, G.H., Littlewood, J.E., Pólya, G.: Inequalities. Cambridge University Press, Cambridge (1934)

6. Hong, Y.: On Hardy-Hilbert integral inequalities with some parameters. J. Inequal. Pure Appl. Math. 6(4), 1-10 (2005). Art. 92

7. Huang, Q.L., Yang, B.C.: A more accurate Hardy-Hilbert-type inequality. J. Guangdong Univ. Educ. 35(5), 27-35 (2015)

8. Krnić, M., Pečarić, J.E.: Hilbert's inequalities and their reverses. Publ. Math. Debr. 67(3-4), 315-331 (2005) 
9. Krnić, M., Pečarić, J.E., Vuković, P.: On some higher-dimensional Hilbert's and Hardy-Hilbert's type integral inequalities with parameters. Math. Inequal. Appl. 11, 701-716 (2008)

10. Krnić, M., Vuković, P.: On a multidimensional version of the Hilbert-type inequality. Anal. Math. 38, 291-303 (2012)

11. Kuang, J.C.: Applied Inequalities. Shangdong Science Technic Press, Jinan (2004)

12. Kuang, J.C., Debnath, L.: On Hilbert's type inequalities on the weighted Orlicz spaces. Pac. J. Appl. Math. 1(1), 95-103 (2007)

13. Kuang, J.C.: Real Analysis and Functional Analysis. Higher Education Press, Beijing (2014)

14. Liu, Q.: A Hilbert-type integral inequality under configuring free power and its applications. J. Inequal. Appl. 2019, 91 (2019)

15. Mitrinović, D.S., Pečarič, J.E., Fink, A.M.: Inequalities Involving Functions and Their Integrals and Derivatives. Kluwer Academic Publishers, Boston (1991)

16. Rassias, M.Th., Yang, B.C.: On half-discrete Hilbert's inequality. Appl. Math. Comput. 220, 75-93 (2013)

17. Rassias, M.Th., Yang, B.C.: A multidimensional half-discrete Hilbert-type inequality and the Riemann zeta function. Appl. Math. Comput. 225, 263-277 (2013)

18. Rassias, M.Th., Yang, B.C.: On a multidimensional Hilbert-type integral inequality associated to the gamma function. Appl. Math. Comput. 249, 408-418 (2014)

19. Rassias, M.Th., Yang, B.C.: On a multidimensional half-discrete Hilbert-type inequality related to the hyperbolic cotangent function. Appl. Math. Comput. 242, 800-813 (2014)

20. Rassias, M.Th., Yang, B.C.: A half-discrete Hilbert-type inequality in the whole plane related to the Riemann zeta function. Appl. Anal. 97(9), 1505-1525 (2018). https://doi.org/10.1080/00036811. 2017.1313411

21. Rassias, M.Th., Yang, B.C.: Equivalent conditions of a Hardy-type integral inequality related to the extended Riemann zeta function. Adv. Oper. Theory 2(3), 237-256 (2017)

22. Rassias, M.Th., Yang, B.C.: On a Hilbert-type integral inequality in the whole plane related to the extended Riemann zeta function. Complex Anal. Oper. Theory 13(4), 1765-1782 (2018). https://doi. org/10.1007/s11785-018-0830-5

23. Rassias, M.Th., Yang, B.C.: On a Hilbert-type integral inequality related to the extended Hurwitz zeta function in the whole plane. Acta Appl. Math. 160, 67-80 (2019). https://doi.org/10.1007/ s10440-018-0195-9

24. Rassias, M.Th., Yang, B.C.: Equivalent properties of a Hilbert-type integral inequality with the best constant factor related the Hurwitz zeta function. Ann. Funct. Anal. 9(2), 282-295 (2018)

25. Rassias, M.Th., Yang, B.C., Raigorodskii, A.: Two kinds of the reverse Hardy-type integral inequalities with the equivalent forms related to the extended Riemann zeta function. Appl. Anal. Discret. Math. 12, 273-296 (2018)

26. Rassias, M.Th., Yang, B.C.: On an equivalent property of a reverse Hilbert-type integral inequality related to the extended Hurwitz-zeta function. J. Math. Inequal. 13(2), 315-334 (2019)

27. Rassias, M.Th., Yang, B.C.: A reverse Mulholland-type inequality in the whole plane with multiparameters. Appl. Anal. Discret. Math. 13, 290-308 (2019)

28. Rassias, M.Th., Yang, B.C., Raigorodskii, A.: On Hardy-type integral inequality in the whole plane related to the extended Hurwitz-zeta function. J. Inequal. Appl. 2020, 94 (2020)

29. Rassias, M.Th., Yang, B.C., Raigorodskii, A.: On the reverse Hardy-type integral inequalities in the whole plane with the extended Riemann-zeta function. J. Math. Inequal. 14(2), 525-546 (2020)

30. Shi, Y.P., Yang, B.C.: On a multidimensional Hilbert-type inequality with parameters. J. Inequal. Appl. 2015, 371 (2015)

31. Shi, Y.P., Yang, B.C.: A new Hardy-Hilbert-type inequality with multiparameters and a best possible constant factor. J. Inequal. Appl. 2015, 380 (2015)

32. Wang, Z.X., Guo, D.R.: Introduction to Special Functions. Science Press, Beijing (1979)

33. Wang, A.Z., Huang, Q.L., Yang, B.C.: A strengthened Mulholland-type inequality with parameters. J. Inequal. Appl. 2015, 329 (2015)

34. Yang, B.C.: On Hilbert's integral inequality. J. Math. Anal. Appl. 220, 778-785 (1998)

35. Yang, B.C.: Hilbert-Type Integral Inequalities. Bentham Science Publishers Ltd., Sharjah (2009)

36. Yang, B.C.: The Norm of Operator and Hilbert-Type Inequalities. Science Press, Beijing (2009)

37. Yang, B.C.: Discrete Hilbert-Type Inequalities. Bentham Science Publishers Ltd., Sharjah (2011)

38. Yang, B.C.: Two Types of Multiple Half-Discrete Hilbert-Type Inequalities. Lambert Academic Publishing, Saarbrïcken (2012) 
39. Yang, B.C.: An extension of a Hardy-Hilbert-type inequality. J. Guangdong Univ. Educ. 35(3), 1-7 (2015)

40. Yang, B.C., Chen, Q.: On a Hardy-Hilbert-type inequality with parameters. J. Inequal. Appl. 2015, 339 (2015)

41. Yang, B.C., Krnić, M.: On the norm of a multi-dimensional Hilbert-type operator. Sarajevo J. Math. 7(20), 223-243 (2011)

42. Yang, B.C., Rassias, Th.M.: On the way of weight coefficient and research for Hilbert-type inequalities. Math. Inequal. Appl. 6(4), 625-658 (2003)

43. Yang, B.C., Rassias, Th.M.: On a Hilbert-type integral inequality in the subinterval and its operator expression. Banach J. Math. Anal. 4(2), 100-110 (2010)

44. Yang, B.C., Brnetić, I., Krnić, M., Pečarić, J.E.: Generalization of Hilbert and Hardy-Hilbert integral inequalities. Math. Inequal. Appl. 8(2), 259-272 (2005)

45. You, M.H., Guan, Y.: On a Hilbert-type integral inequality with non-homogeneous kernel of mixed hyperbolic functions. J. Math. Inequal. 13(4), 1197-1208 (2019)

46. Zhao, C.Z., Cheung, W.S.: On Hilbert's inequalities with alternating signs. J. Math. Inequal. 12(1), 191-200 (2018)

47. Zhong, W.Y.: The Hilbert-type integral inequality with a homogeneous kernel of Lambda-degree. J. Inequal. Appl. 917392 (2008). https://doi.org/10.1155/2008/917392

48. Zhong, W.Y., Yang, B.C.: On multiple Hardy-Hilbert's integral inequality with kernel. J. Inequal. Appl. 2007 (2007). https://doi.org/10.1155/2007/27. Art.ID 27962

\section{Affiliations}

\section{Michael Th. Rassias ${ }^{1,2,3} \cdot$ Bicheng Yang ${ }^{4} \cdot$ Andrei Raigorodskii $^{2,5,6,7}$}

Bicheng Yang

bcyang@gdei.edu.cn; bcyang818@163.com

Andrei Raigorodskii

mraigor@yandex.ru

1 Institute of Mathematics, University of Zurich, 8057 Zurich, Switzerland

2 Moscow Institute of Physics and Technology, Institutskiy per, d. 9, 141700 Dolgoprudny, Russia

3 Program in Interdisciplinary Studies, Institute for Advanced Study, 1 Einstein Dr, Princeton, NJ 08540, USA

4 Department of Mathematics, Guangdong University of Education, Guangzhou 510303, Guangdong, People's Republic of China

5 Moscow State University, 119991 Moscow, Russia

6 Buryat State University, Ulan-Ude, Russia

7 Caucasus Mathematical Center, Adyghe State University, Maykop, Russia 